CHAPITRE 3

\title{
Le cahier des charges des réacteurs à neutrons rapides du futur
}

François Gauché

CEA 



\section{| Introduction}

La conception, la construction, l'exploitation et le démantèlement d'un réacteur nucléaire doivent répondre à de très nombreux critères. Le présent chapitre ne saurait remplacer un cahier des charges détaillé. Au moment où I'on s'intéresse aux réacteurs du futur, dits de IV e génération, et en particulier aux réacteurs à neutrons rapides (RNR), l'objectif est d'une part de montrer la grande diversité des contraintes à prendre en compte, qui distinguent du réacteur « papier » le réacteur réel qui produira un jour de l'électricité, et qui pour la majorité sont des challenges scientifiques et technologiques qui ne se voient pas forcément au premier coup d'œil. D'autre part, s'agissant de RNR de nouvelle génération, on s'intéressera à de nouvelles contraintes qui découlent de l'expérience passée et de l'accroissement des exigences, de sûreté en particulier, qui pèsent sur la conception de tout nouveau réacteur nucléaire.

Le présent chapitre s'alimente du cahier des charges pour les futurs réacteurs à neutrons rapides refroidis au sodium réalisé par EDF et transmis au CEA dans le cadre du projet ASTRID ${ }^{1}$. II s'en éloigne parfois, en particulier pour tenir compte des autres types de réfrigérants abordés au cours du séminaire «Fluides caloporteurs pour réacteurs à neutrons rapides » organisé par l'Académie des sciences les 19 et 20 février 2013.

\section{Attentes des systèmes de $\mathrm{IV}^{\mathrm{e}}$ génération}

\subsection{Le forum international génération IV}

Le cadre international de la coopération en matière de systèmes nucléaires de $4^{\mathrm{e}}$ génération est le GIF (Generation IV International Forum), dont I'objectif est la conduite des travaux de recherche et développement (R\&D) nécessaires à la mise au point de systèmes nucléaires (réacteurs et cycle du combustible) répondant aux critères de durabilité de l'énergie nucléaire : poursuite des progrès en compétitivité et en sûreté atteints sur les réacteurs à eau de $3^{\mathrm{e}}$ génération, économie des ressources, minimisation de la production des déchets radioactifs, plus grande résistance à la prolifération nucléaire, application de l'énergie nucléaire à d'autres voies que la production d'électricité.

Le GIF est une association intergouvernementale lancée en 2000 à l'initiative du département de l'Énergie des États-Unis. II regroupe à ce jour treize

1 Advanced Sodium Technological Reactor for Industrial Demonstration. 
membres $^{2}$ engagés par la signature d'une charte, en juillet 2001, dans laquelle ils reconnaissent l'importance du développement de systèmes futurs pour la production d'énergie nucléaire, ainsi que la nécessité à la fois de préserver au mieux l'environnement et de se prémunir contre les risques de prolifération.

Un accord cadre intergouvernemental consolidant ces engagements a été signé à partir de février 2005 par dix membres, dont la France. L'Argentine et le Brésil ont souhaité différer leur signature et demeurent provisoirement dans un statut de membre non actif. Le Royaume-Uni n'a pas ratifié l'accord cadre signé en 2005, préférant limiter à ce stade sa contribution au programme européen. Cet accord fixe le cadre des accords juridiques plus détaillés permettant une coopération équitable entre partenaires. Chaque membre du GIF est représenté par un ou plusieurs agents de mise en application des objectifs de l'accord cadre.

\subsection{Les six concepts du GIF}

Le Forum international génération IV a sélectionné six concepts paraissant les plus prometteurs et a défini un plan de R\&D visant à apporter les innovations nécessaires pour le déploiement industriel, à partir de 2030, des systèmes basés sur ces concepts. Le plan de développement de ces six systèmes comprend trois phases : faisabilité, performances et démonstrations. L'accord cadre et les accords détaillés qui en découlent ne couvrent que les deux premières phases. La réalisation de démonstrations, pilotes ou prototypes nécessitent des accords différents.

Les concepts sélectionnés par le GIF sont les suivants :

- concepts à neutrons rapides : réacteur à neutrons rapides refroidi au sodium (RNR-Na, en anglais Sodium-cooled Fast Reactor SFR), réacteur à neutrons rapides refroidi par gaz (RNR-G, Gas-cooled Fast Reactor GFR), réacteur à sels fondus (RSF, Molten Salt (Fast) Reactor M(F)SR), réacteur à neutrons rapides refroidi au plomb (RNR-Pb, Lead-cooled Fast Reactor LFR) ;

- concepts à neutrons thermiques : réacteur refroidi par eau-supercritique (RESC, Super Critical Water-cooled Reactor SCWR), réacteur à très haute température (RTHT, Very High Temperature Reactor VHTR).

2 Afrique du Sud, Argentine, Brésil, Canada, Chine, États-Unis, Euratom, France, Japon, République de Corée du Sud, Royaume-Uni, Russie, Suisse. 
II faut noter que la maturité technologique des concepts retenus par le GIF est très variable. Pour le CEA, l'effort se concentre principalement sur les technologies de réacteurs à neutrons rapides refroidis au sodium et, dans une moindre mesure, surtout en ce qui concerne l'innovation sur les matériaux, sur les technologies de refroidissement par gaz, dans une vision à beaucoup plus long terme. La R\&D au niveau français est principalement portée par les équipes du CEA, d'AREVA et d'EDF. Le CNRS travaille sur un concept de réacteur rapide à sels fondus.

\subsection{Objectifs des systèmes de IV ${ }^{\mathrm{e}}$ génération}

Les objectifs fixés pour les systèmes de IV ${ }^{e}$ génération sont les suivants :

- pouvoir recycler sans limitation les matières comme le plutonium et utiliser le mieux possible la ressource en uranium (ou thorium). Ceci nécessite des réacteurs fonctionnant en spectre de neutrons rapides, couplés à un cycle fermé du combustible. Rappelons que l'utilisation dans des réacteurs à neutrons rapides de la ressource en uranium appauvri déjà extrait et disponible sur le territoire français permettrait de produire de l'électricité au rythme actuel pendant plusieurs milliers d'années ;

- si cette option est retenue, avoir la capacité de poursuivre les études nécessaires pour réaliser la transmutation de certains actinides mineurs. De la même façon, ceci nécessite des réacteurs à neutrons rapides ;

- avoir un niveau de sûreté correspondant au meilleur standard du moment. Ceci équivaut aujourd'hui aux objectifs de sûreté des réacteurs de III ${ }^{\mathrm{e}}$ génération auxquels il faut intégrer dès la conception les enseignements de l'accident de Fukushima, en particulier la recherche d'une démonstration de sûreté robuste, prenant en compte les incertitudes et garantissant suffisamment de marge par rapport aux « effets falaise ${ }^{3}$;

- atteindre une bonne compétitivité compte tenu du service rendu ;

- présenter des garanties de résistance à la prolifération nucléaire.

3 Effet falaise : altération brutale du comportement d'une installation, que suffit à provoquer une légère modification du scénario envisagé pour un accident dont les conséquences sont alors fortement aggravées. 


\section{Attentes en termes de performances}

Même s'il doit obéir à un certain nombre de contraintes, de sûreté en particulier, un réacteur nucléaire est construit, la plupart du temps, avec un objectif économique, généralement celui de produire de l'électricité. S'ajoutent à cela les exigences de sécurité d'approvisionnement et d'indépendance énergétique. L'exploitant de la centrale et la collectivité attendent donc du réacteur des performances en matière de production. Dans le cas des réacteurs à neutrons rapides, le présent chapitre commente quelques-unes de ces spécifications à la lumière du choix du fluide caloporteur.

\subsection{Puissance du réacteur}

Quand le réseau électrique le permet, et si l'on met de côté les réflexions actuelles sur les petits réacteurs modulaires (small modular reactors) et leur modèle économique particulier, la plupart des calculs économiques poussent à rechercher des réacteurs nucléaires de grande taille, d'au moins $1000 \mathrm{MWe}$, en raison des effets d'échelle qui permettent en particulier d'accommoder plus facilement les surcoûts des nouvelles exigences de sûreté. Ajouter à cela les avantages des « effets de série » qui sont un atout majeur pour les coûts de fabrication, la maintenance, le retour d'expérience d'exploitation et l'amélioration continue de la sûreté. S'agissant de premières unités déployées à des fins de démonstration ou de début de série, on peut accepter des réacteurs de plus petite taille. Ceci permet de fixer deux plages de puissance, qui ne sont données que de manière indicative :

- gamme 1000-2500 MWth pour les premières unités ;

- gamme 3000-4000 MWth pour un déploiement industriel massif.

Il faut noter que ces gammes de puissance ne sont pas forcément accessibles avec tous les fluides caloporteurs. Par exemple, on peut être amené à limiter la puissance des réacteurs refroidis par le plomb ou l'eutectique plombbismuth en raison de la taille des cœurs et de la masse du circuit primaire. En effet, pour éviter la corrosion-érosion par le plomb, une des stratégies consiste à limiter à $2 \mathrm{~m} / \mathrm{s}$ la vitesse de circulation du caloporteur, ce qui oblige à écarter les assemblages. Ceci conduit pour les fortes puissances à des cœurs très grands dont l'effet de vide est très fortement positif (la perte du caloporteur se traduit par une forte excursion de puissance). Par ailleurs, les exigences de tenue au séisme pour des grandes tailles de circuit primaire sont particulièrement contraignantes dans le cas d'un caloporteur dont la densité est plus de 10 fois celle du sodium ou de l'eau. 


\subsection{Gain de régénération}

L'une des caractéristiques essentielles des réacteurs à neutrons rapides est leur capacité à pouvoir atteindre la surgénération, c'est-à-dire, à partir de matières fertiles comme l'uranium-238 ou le thorium-232, à produire plus de matière fissile qu'ils n'en consomment (gain de régénération $G R>1$ ). Mais ces réacteurs peuvent aussi fonctionner en mode isogénérateur (équilibre production/consommation de matières fissiles; $G R \sim 11$ ou même sous-générateur (consommation nette de matières fissiles; $G R<1$ ). On parle parfois de mode " brûleur » pour désigner un réacteur conçu spécialement pour consommer de façon accrue le plutonium.

Tout dépend donc de la raison pour laquelle on cherche à déployer des réacteurs à neutrons rapides. Ceux-ci ont besoin d'une quantité de matière fissile au démarrage. Historiquement, on a donc considéré des surgénérateurs, capables de produire la quantité de plutonium nécessaire au démarrage de réacteurs supplémentaires de façon à permettre la croissance d'un parc de RNR qui, une fois démarrés, ne consomment que très peu de ressources en uranium. C'est cette hypothèse qui est retenue par des pays dont les besoins énergétiques sont en forte croissance, comme la Chine ou l'Inde.

Dans d'autres pays avec un parc de réacteurs à eau légère fonctionnant depuis plusieurs décennies comme la France, la quantité de plutonium requise pour démarrer un parc de RNR n'est pas forcément le facteur limitant. Dans ce cas, d'autres considérations peuvent amener à préférer des réacteurs isogénérateurs associés à l'objectif de stabiliser la quantité de plutonium dans le cycle.

$\mathrm{Si}$ l'on cherche à réduire la quantité de plutonium dans le cycle, des concepts sous-générateurs ou brûleurs peuvent être recherchés.

Il faut noter que la combinaison des choix du combustible, du caloporteur et du concept de cœur peut restreindre la plage de gains de régénération qui sont atteignables. Par exemple, le concept de cœur à faible vidange développé par le CEA pour le projet ASTRID ne permet pas d'atteindre des valeurs de gain de régénération très élevées (limite à 1,15 environ), alors que certains pays affichent l'ambition de gains de régénération de 1,3 voire 1,4. En revanche, on peut développer un concept CFV «brûleur » avec de bonnes performances de consommation nette de plutonium.

Enfin, la question des couvertures fertiles radiales est un sujet controversé, dans la mesure où une certaine littérature les considère comme plus faciles à détourner à des fins de production de matière fissile militaire. L'argument étant que dans des couvertures radiales, le vecteur isotopique du plutonium se rapproche de celui des applications militaires. Or, selon les concepts, 
I'isogénération ou la surgénération ne peuvent être atteintes qu'avec des couvertures radiales. Pour ASTRID, on s'est fixé au contraire comme objectif $d$ 'atteindre l'isogénération sans couvertures radiales.

\subsection{Durée de vie}

À l'image des réacteurs de $\|{ }^{\mathrm{e}}$ génération, on attend des réacteurs à neutrons rapides de $I V^{e}$ génération de pouvoir justifier d'une durée de vie d'au moins 60 ans. Ayant affaire à des conditions de fonctionnement particulièrement contraignantes (températures élevées, possible corrosion, cycles...), ceci nécessite pour les composants non remplaçables une $R \& D$ de long terme sur les matériaux, afin de disposer des bases de données sur une échelle de temps suffisamment représentative pour pouvoir extrapoler à une durée de vie de 60 ans. Certains composants pour lesquels cette durée de vie ne sera pas démontrée devront être conçus pour être remplaçables (générateurs de vapeur par exemple).

Tous les caloporteurs ne partent pas à égalité sur ce sujet. Concernant le RNR-Na, plusieurs décennies de fonctionnement ont permis d'accumuler un patrimoine de connaissances considérable sur les matériaux et leur comportement en réacteur. Certains échantillons issus du démantèlement des réacteurs, en particulier Phénix, viendront alimenter les dossiers de justification de la durée de vie des futures centrales de ce type.

\subsection{Taux de combustion, longueur des cycles}

Pour améliorer la disponibilité des réacteurs, il faut viser les cycles les plus longs possibles, séparés par des arrêts pour rechargement les plus courts possibles. Ce raisonnement ne s'applique bien évidemment pas aux réacteurs à sels fondus.

On recherche donc pour la filière industrielle des taux de combustion élevés, de l'ordre de 180 à $200 \mathrm{GWj} / \mathrm{t}$, avec des cycles de 18 à 24 mois. Ceci impose un programme de $R \& D$ et de qualification en réacteur pour l'amélioration des performances des combustibles, sachant que pour le RNR-Na, de grands progrès ont été faits dans le passé en passant de quelques dizaines de $\mathrm{GWj} / \mathrm{t}$ à plus de $100 \mathrm{GWj} / \mathrm{t}$.

Selon les concepts de cœur et le choix du caloporteur, on recherche des alliages à faible gonflement sous irradiation, comme les aciers renforcés par dispersion d'oxyde sur lesquels un important effort de R\&D est réalisé dans le 
monde. En effet, si l'on prend le cas du caloporteur sodium, les assemblages combustibles sont faits d'aiguilles de combustible (l'équivalent des crayons de combustible dans un REP) séparés par des fils métalliques enroulés autour de l'aiguille. L'espace entre les aiguilles est très réduit (entre 1 et $2 \mathrm{~mm}$ ) et un gonflement des gaines viendrait réduire cet espace et réduire les échanges thermiques.

\subsection{Taux de disponibilité}

On vise un taux de disponibilité supérieur à $90 \%$. Pour les prototypes, la disponibilité visée reste ambitieuse, mais le calcul devrait déduire les indisponibilités qui seront dues aux diverses démonstrations et expérimentations qui seront réalisées dans le réacteur.

Le taux de disponibilité dépend en particulier de la fréquence des arrêts pour rechargement (voir le point précédent) et de leur durée. Celle-ci est reliée aux opérations de passage du fonctionnement vers l'arrêt pour manutention, à la manutention des assemblages usés et neufs, et au redémarrage. Il y a aussi les inspections périodiques des équipements et la requalification des systèmes importants pour la sûreté, par exemple les mécanismes de barre de commande.

La disponibilité peut aussi être grandement affectée par des opérations de réparation ou remplacement de composants.

Citons enfin les délais liés aux autorisations administratives, en particulier pour les arrêts non programmés.

\subsection{Coûts}

La question de la compétitivité des réacteurs de IVe génération par rapport aux autres sources d'énergie ne se résume pas simplement et renvoie à des débats complexes qui analysent les rapports coûts/bénéfices en détail et dépassent le cadre de cet article.

Il y a fort à parier que les réacteurs à neutrons rapides resteront, à l'investissement, plus chers que des réacteurs à caloporteur eau. Cela vient du fait que les fluides caloporteurs autres que l'eau sont plus compliqués à gérer que ce fluide courant. II s'agit donc de maintenir le surcoût à l'investissement aussi bas que possible, sachant que, selon les hypothèses que l'on émet sur l'évolution des prix de l'uranium, ce surcoût peut être in fine compensé grâce à l'avantage des neutrons rapides sur le cycle des matières. 
Avant l'atteinte d'un coût optimisé après remise en route du tissu industriel et construction de plusieurs unités, il faut avoir à l'esprit les surcoûts inhérents à l'étape du prototype ou de la tête de série.

Enfin, les réacteurs à neutrons rapides remplissent des fonctions supplémentaires par rapport aux réacteurs à eau légère. Ces fonctions, qui concernent le cycle des matières nucléaires, n'ont pas forcément une valeur économique facilement mesurable, mais correspondent à un service qui peut être de nature stratégique. La stabilisation des stocks de plutonium, la sécurisation de l'approvisionnement énergétique, l'indépendance par rapport aux mines d'uranium, la possible incinération de certains actinides mineurs... peuvent être des atouts valorisables.

\section{Attentes en termes de sûreté}

\subsection{Quel niveau de sûreté ?}

II n'est pas possible de définir précisément le niveau de sûreté attendu pour les futures générations de réacteurs nucléaires, compte tenu des incertitudes, d'une part sur la date à laquelle ces réacteurs seront déployés (2040? 2080 ?....), d'autre part sur les évolutions des attentes de la société en matière de sûreté, reprises par les autorités de sûreté nucléaire, entre aujourd'hui et cette date de déploiement.

Ainsi, au-delà d'une recherche continue d'amélioration de la sûreté, le niveau de sûreté requis a progressé dans le passé par paliers successifs, en particulier après les accidents de Three Mile Island, Tchernobyl et Fukushima, et après les attentats du World Trade Center en 2001.

Le référentiel de sûreté, mais aussi de radioprotection, de rejets dans l'environnement, etc., qui découle de ces évènements et de plusieurs décennies d'exploitation de réacteurs nucléaires dans le monde est donc devenu très exigeant, sans commune mesure avec les règles des temps pionniers au lendemain de la Seconde Guerre mondiale, où de nombreuses technologies ont pu être testées. C'est dire l'effort à faire pour amener au niveau de sûreté requis un concept ne bénéficiant d'aucun retour d'expérience.

Si l'on regarde l'étape des prototypes qui pourraient être mis en service dans la prochaine décennie, on peut proposer un niveau de sûreté équivalent à un réacteur à eau légère de $1 / \mathrm{I}^{\mathrm{e}}$ génération (document WENRA ${ }^{4}$ Safety Objectives for New Nuclear Power Plants, 2010), doublé de l'exigence d'avoir intégré

\footnotetext{
4 Western European Nuclear Regulators' Association.
} 
convenablement le retour d'expérience des réacteurs antérieurs, et prenant en compte dès la conception les leçons tirées du retour d'expérience de l'accident de Fukushima, en particulier l'exclusion d'effet falaise dès qu'une agression externe dépasse un peu les limites du domaine de dimensionnement.

En particulier, I'objectif $\mathrm{O}$ du document WENRA cité au-dessus requiert :

- que la conception permette d'exclure les accidents conduisant à des rejets précoces ou importants ;

- qu'en cas de fusion du cœur, des dispositions de conception permettent de limiter dans le temps et dans l'espace les mesures de protection des populations et que les délais soient suffisants pour les mettre en œuvre.

À la lumière de cet objectif 03 , la réalisation d'études probabilistes de sûreté de niveau 2 en complément de la démonstration de sûreté probabiliste est particulièrement pertinente. En effet, ces études de niveau 2 vont jusqu'à la quantification des rejets à l'extérieur de la centrale et donnent un éclairage supplémentaire par rapport à la démonstration déterministe.

\subsection{Principales fonctions de sûreté}

L'ordre dans lequel ces fonctions vont être déclinées ne préjuge en rien de leur importance relative. Toutes sont cruciales. En cas d'incident, l'arrêt de la réaction en chaîne constitue la première fonction de sûreté. Ceci conduit à concevoir des systèmes d'arrêt extrêmement fiables, redondants et diversifiés. À noter la particularité des RNR-Pb (barres d'arrêt insérées par le bas par la force de flottaison dans le plomb ou de façon pneumatique par le haut) et des réacteurs à sels fondus (pas de barres d'arrêt).

Ensuite, l'évacuation de la puissance résiduelle constitue un enjeu majeur. II s'agit d'exclure la perte totale de cette fonction sur une longue période de temps. La combinaison de systèmes actifs et passifs, redondants et diversifiés, permet $d$ 'atteindre cet objectif. II faut toutefois faire particulièrement attention au risque de gel du caloporteur dans les circuits, conduisant à la perte du circuit.

Vis-à-vis de l'évacuation de la puissance résiduelle, le caloporteur gaz a l'inconvénient d'une faible capacité calorifique, ce qui conduit à devoir se protéger des accidents de dépressurisation.

La troisième fonction de sûreté est le confinement. Notons qu'elle doit être complètement repensée dans le cas du réacteur à sels fondus, dans la mesure où le combustible ne dispose pas de sa première barrière habituelle qui est la gaine. 
Enfin, citons quatre enjeux liés au choix du caloporteur :

- la compatibilité du caloporteur primaire avec le caloporteur secondaire. Pour de nombreuses raisons, on préfère séparer la partie conventionnelle de l'installation (turbine) du circuit primaire. On a donc au moins un circuit secondaire, parfois même un troisième circuit (cas du sodium et des sels fondus). Il faut donc tenir compte d'une possible mise en contact des fluides primaire et secondaire. C'est en particulier la problématique des réactions sodium-eau qui oblige à avoir un circuit intermédiaire en sodium pour les réacteurs de ce type. Mais les interactions peuvent aussi conduire à l'entraînement de gaz dans le cœur (et à un accroissement de la réactivité), à la formation de précipités solides, à une explosion de vapeur... ;

- la purification. L'enjeu est en particulier d'éviter la corrosion des structures, ainsi que la formation de composés solides qui viendraient boucher des circuits ou des assemblages. Cette problématique est particulièrement importante pour les caloporteurs plomb et plomb-bismuth ;

- dans le cas du sodium, les grandes réactions sodium-eau, sodium-eauair et les grands feux sodium. L'objectif de la conception sera d'éliminer de telles possibilités ou d'en confiner les conséquences ;

- la détection de fuites : la fuite du caloporteur est le signe d'une défaillance des circuits. Elle doit être détectée au plus tôt pour éviter une dégradation de la situation (corrosion sous calorifuge, feu de sodium, rupture de tuyauterie, contamination radioactive par du sel fondu, accident de dépressurisation ou baisse de niveau primaire...).

\subsection{Agressions externes, états de I'installation}

Les agressions externes peuvent être d'origine humaine (chute d'avion), malveillante ou non, ou d'origine naturelle. Nous allons examiner successivement les deux cas.

Concernant la chute d'avion, il y a souvent confusion entre la prise en compte d'un risque probabiliste, à savoir la chute accidentelle d'un aéronef, et l'acte de malveillance (chute volontaire). Il convient de protéger la centrale contre les deux éventualités, sachant que le référentiel concernant la malveillance est confidentiel pour des raisons évidentes. Le choix du caloporteur $n^{\prime}$ est pas neutre face à ce type d'agression externe. Citons par exemple la densité élevée du plomb qui rendra difficile la conception d'une grande cuve à coque mince, ou bien le sodium des circuits intermédiaires et des bâtiments des générateurs de vapeur situés à l'extérieur du bâtiment réacteur. 
Le séisme doit également être pris en compte, en suivant les règles fondamentales de sûreté ou les guides émis par l'Autorité de sûreté nucléaire. Là aussi, le caloporteur plomb impose aux concepteurs des précautions particulières.

La prise en compte du risque d'inondation interdira bien sûr de disposer des réservoirs de sodium dans des locaux inondables. Certains événements externes, comme l'incendie de forêt, devront être analysés par rapport au fonctionnement d'échangeurs de chaleur avec l'atmosphère, utilisés pour l'évacuation de la puissance résiduelle (risque de colmatage). Les grands froids devront être regardés aussi (risque de gel, etc.).

Sur un autre plan, il faut noter qu'une démarche de sûreté moderne prendra en compte toutes les parties de l'installation et tous les états de fonctionnement. Par exemple, l'accident de Fukushima a montré à quel point il faut accorder à l'entreposage de combustibles usés la même protection qu'au réacteur lui-même. Des analyses de sûreté spécifiques seront ainsi nécessaires pour, entre autres, les cuves ou les piscines d'entreposage de combustible, les réservoirs de sels fondus primaires ou les circuits du procédé de traitement en ligne du sel fondu.

\subsection{Radioprotection, déchets, démantèlement}

La conception de type intégré des réacteurs à caloporteur sodium a montré dans le passé qu'une dosimétrie collective aux travailleurs beaucoup plus basse que sur les REP était possible. De manière indicative, on peut proposer un objectif de dose annuelle collective aux travailleurs de moins de 100 hommes.mSv par an et par GWe installé, ce qui correspond environ à un dixième de la dose sur un REP de $\|{ }^{e}$ génération. En optimisant certaines opérations de maintenance dès la conception, on peut également limiter la dose individuelle à $2,5 \mathrm{mSv} / \mathrm{an}$, ce qui la ramène au niveau de la radioactivité naturelle en France.

À noter le défi, pour les concepteurs de réacteurs à sels fondus, de prévoir dès le début la maintenance et l'éventuel remplacement des composants contaminés par le sel primaire, dans le respect de l'objectif de minimiser la dose reçue par les travailleurs.

On peut aussi signaler comme exemple la problématique du polonium-210, très radiotoxique, produit par activation du bismuth dans le fluide primaire des réacteurs refroidis au plomb ou plomb-bismuth.

Concernant la production de déchets, on préférera les déchets solides. Enfin, la question du tritium, qui est produit dans le réacteur habituellement dans le combustible ou dans les absorbants, prend une dimension nouvelle 
avec les caloporteurs à base de sels de lithium et doit faire l'objet de dispositions de conception adaptées (équipements de détritiation).

Le retour d'expérience de démantèlement d'installations nucléaires, y compris de quelques réacteurs à neutrons rapides refroidis au sodium, permet de réfléchir dès la conception à la simplification des procédures de démantèlement, à la minimisation des déchets produits et de la dose aux travailleurs. La disponibilité des filières d'évacuation est également prise en compte.

Par exemple, on cherchera à remplacer les stellites par des revêtements durs ne contenant pas du cobalt, ce qui diminue fortement l'activation des structures du réacteur. Mais les solutions de remplacement dépendent évidemment de la compatibilité du revêtement avec le caloporteur, et seront donc variables selon les concepts.

Le démantèlement est d'ailleurs un des défis des réacteurs à sels fondus dans la mesure où, par conception, c'est l'ensemble du circuit primaire qui est contaminé par les actinides et les produits de fission.

\section{Quelques enjeux spécifiques des réacteurs à neutrons rapides}

\subsection{Cœur}

Les cœurs des réacteurs à neutrons rapides sont sensibles en général à l'« effet de vide » du caloporteur, c'est-à-dire que si le caloporteur disparaît localement, par ébullition par exemple, la réactivité du réacteur augmente. Ceci n'est évidemment pas le cas pour les sels fondus. L'effet est très faible dans le cas du caloporteur gaz. II reste important pour les grands cœurs des réacteurs à caloporteur plomb et sodium. Pour le plomb, on peut mettre en avant que la température d'ébullition est très élevée $\left(1749^{\circ} \mathrm{C}\right)$. Pour le sodium, les études sur le prototype ASTRID ont permis de développer un concept de cœur à faible effet de vidange sur la réactivité, pour lequel le coefficient correspondant est négatif au lieu d'être fortement positif pour les conceptions classiques. De nombreuses études sont encore nécessaires dans les prochaines années pour quantifier et réduire les incertitudes, ce qui passe en particulier par la réalisation d'expériences dans des réacteurs à puissance nulle (" maquettes critiques »), qui permettent de valider les calculs neutroniques.

Par ailleurs, les cœurs de réacteurs à neutrons rapides ne fonctionnent pas dans leur géométrie la plus réactive et sont donc sensibles aux phénomènes de compaction. Des dispositions constructives permettant de garantir les jeux entre assemblages et d'exclure les cas où la prompt-criticité pourrait être atteinte (durant le transitoire) sont nécessaires sur les concepts SFR, LFR et 
GFR. Mais les possibilités de scénarios de compaction devront être analysées de près également pour les réacteurs à sels fondus (stabilité du sel, homogénéité, distribution des gaz dissous...).

Les événements dits locaux doivent également être pris en compte, comme la remontée intempestive d'une barre de commande ou le bouchage d'un assemblage, dans la mesure où, sous certaines conditions, ils peuvent être à l'origine de fusion généralisée du cœur. La détection de rupture de gaine fait partie des lignes de défense, associée au concept de « cœur propre » qui interdit de fonctionner avec des ruptures de gaine.

La prise en compte de la possibilité d'une fusion du cœur conduit à étudier les interactions entre le corium et le caloporteur sodium ou plomb, avec l'éventualité d'un dégagement d'énergie mécanique qui vienne solliciter la limite du circuit primaire, qui doit donc être conçu avec une certaine résistance. La validation des codes de calcul dans ce domaine requiert des expérimentations difficiles à réaliser (nécessité de préparer à plus de $2000^{\circ} \mathrm{C}$ un corium expérimental de taille suffisamment représentative).

L'accident grave devant être couvert par le dimensionnement de l'installation, il faut se préoccuper du devenir du corium et de son refroidissement sur le long terme. On peut concevoir ainsi pour le SFR ou le GFR un récupérateur de corium à l'instar de ce qui est fait sur l'EPR. La question se pose différemment sur le LFR puisque le corium flotte plus ou moins dans le caloporteur. Enfin, il faut donner à l'accident grave, qui correspond classiquement à la fusion du cœur, une définition différente pour le MSR.

\subsection{Manutention du combustible}

L'utilisation de caloporteurs autres que l'eau complexifie la manutention des assemblages combustibles. Citons en particulier la question de savoir si l'on a besoin de pouvoir réaliser, rapidement ou non, le déchargement complet du cœur au cours de la durée de vie du réacteur. Ou bien la prévention des erreurs de manutention, le lavage des assemblages... Le poste manutention peut avoir un impact important sur la disponibilité du réacteur, même pour un réacteur à sels fondus où l'indisponibilité peut venir d'une panne prolongée du système de traitement en ligne des sels.

\subsection{Instrumentation et inspection en service}

Si le caloporteur est opaque, comme le plomb ou le sodium, I'inspection des structures peut devenir un vrai défi alors qu'elle est obligatoire dans 
la démonstration de sûreté. Mais, au-delà de l'opacité, d'autres obstacles peuvent apparaître comme la température (la température d'arrêt à « froid » $d^{\prime}$ un RNR plomb est autour de $400^{\circ} \mathrm{C}$ ) ou le rayonnement qui peut raccourcir très fortement la durée de vie du capteur. Même si des techniques, ultrasonores notamment, ont pu être développées et mises en œuvre avec succès par le passé sur les RNR-Na, l'inspection en service reste un sujet pour lequel de grandes avancées sont encore nécessaires pour atteindre un niveau qui soit considéré comme suffisamment industriel.

L'accident de Fukushima a fait entrer dans le référentiel de conception le besoin de disposer d'une instrumentation post-accidentelle capable de renseigner l'exploitant sur l'état de son installation pendant et après l'accident (localisation du corium par exemple).

\subsection{Disponibilité du caloporteur}

Un des critères intervenant dans le choix du caloporteur est son prix et sa disponibilité. Le sodium et le plomb ne posent pas de problème particulier. La disponibilité du bismuth, seulement deux fois plus abondant que l'or à la surface de la Terre, doit être étudiée de près pour un déploiement industriel. Quant à l'hélium, sa cherté impose une très bonne maîtrise des fuites, et il faut faire attention aux capacités de production qui peuvent avoir un impact sur les prix et la disponibilité.

\section{Conclusion}

Le présent chapitre ne saurait prétendre à l'exhaustivité. On a cherché à illustrer la grande variété d'enjeux en lien avec le choix du caloporteur.

Méfions-nous donc des jugements rapides, basés sur une sélection trop partielle de critères. II n'existe pas de caloporteur idéal qui résolve tous les inconvénients des autres caloporteurs. Le choix du caloporteur ne peut se faire que dans la confrontation du concepteur avec tous les enjeux entrant en ligne de compte, qu'ils soient considérés comme nobles (par exemple la beauté du concept neutronique) ou plus vulgaires (c'est chaud donc on perd beaucoup de temps à la maintenance).

Enfin, il y a indubitablement un lien entre la maturité technologique, le retour d'expérience et la sûreté. Dans un contexte où les exigences de sûreté n'arrêtent pas d'augmenter, il y a de fait une «barrière à l'entrée » pour les concepts basés sur des caloporteurs moins usuels. 